\title{
Neuromodulation in Inflammatory Skin Disease
}

Eric J. Yang · Sahil Sekhon · Kristen M. Beck •

Tina Bhutani $\cdot$ John Koo

Received: December 28, 2017 / Published online: February 9, 2018

(C) The Author(s) 2018. This article is an open access publication

\begin{abstract}
Inflammatory skin diseases are difficult to treat because of a lack of available treatment options for severe disease. However, recent advances have shown that vagus nerve stimulation can be used to decrease inflammation and reduce disease severity in rheumatoid arthritis and inflammatory bowel disease. Changes in cytokine profiles observed in these studies are similar to those seen with use of biologics in inflammatory skin disease, suggesting that they act along similar pathways to disrupt chronic inflammation and treat inflammatory disease. This commentary explores the existing evidence demonstrating the efficacy of neuromodulation in inflammatory disease, and outlines reasons why these findings could translate to the dermatology setting to treat inflammatory skin disease.
\end{abstract}

Enhanced content To view enhanced content for this article go to https://doi.org/10.6084/m9.figshare. 5841042 .

E. J. Yang $(\bowtie) \cdot$ S. Sekhon · K. M. Beck ·

T. Bhutani $\cdot$ J. Koo

Department of Dermatology, University of

California-San Francisco, San Francisco, CA, USA

e-mail: ericjyang@outlook.com

\section{E. J. Yang}

Chicago Medical School, Rosalind Franklin

University of Medicine and Science, North Chicago,

IL, USA
Keywords: Autonomic

imbalance; Inflammation; Inflammatory skin disease; Neuromodulation; Treatment; Vagus nerve stimulation

\section{INTRODUCTION}

Despite significant advances in treatment options for psoriasis and atopic dermatitis, treatment for inflammatory skin diseases as a whole still has much room for improvement. Although minor cases of rosacea, seborrheic dermatitis, lichen planus, hidradenitis suppurativa, and other inflammatory skin diseases can often be treated with topical therapies, severe disease poses a unique challenge for dermatologists as a result of limited treatment options for these disorders.

New advances in the fields of rheumatoid arthritis (RA) and inflammatory bowel disease (IBD) indicate that vagus nerve stimulation (VNS) reduces inflammation and decreases disease severity. The nervous system's intimate relationship with inflammation suggests that neuromodulation may serve as a potential treatment for inflammatory skin disease, an avenue that has hardly been explored in dermatology. 


\section{Immunomodulatory Reflex}

The "immunomodulatory reflex" is a wellcharacterized mechanism that aims to maintain a balanced immune system [1]. The afferent arm of this reflex consists of sensory nerves that detect injury, infection, and inflammation, while the efferent arm consists of the "cholinergic anti-inflammatory pathway". Stimulation of the vagus nerve within the "cholinergic antiinflammatory pathway" results in increased cholinergic outflow, which has been shown to decrease innate immune cell production of cytokines $[1,2]$. This pathway has been shown to play an essential role in decreasing inflammation in various models of peritonitis, pancreatitis, and other inflammatory syndromes [1].

\section{Neuromodulation: A Useful Anti- Inflammatory Intervention}

Various chronic inflammatory diseases demonstrate autonomic dysfunction, including IBD $[3,4]$, RA [5, 6], and psoriatic arthritis (PsA) [7]. In these diseases, sympathetic tone predominates, typically as a result of parasympathetic nervous system dysfunction. Nervous system tone plays an important role in inflammatory disease, as increased autonomic imbalance has been associated with diminished response to anti-inflammatory treatment [8]. Thus, restoration of this balance presents a potential treatment option for inflammatory disease. Initial studies in RA and IBD have demonstrated promising results regarding the anti-inflammatory properties of VNS.

Early studies of neuromodulation showed that treatment using cholinergic agonists prevents development of arthritis in murine models of RA [9, 10]. VNS for 6 days in mouse models for RA were found to significantly decrease inflammation, resulting in decreased bone erosion and cartilage destruction compared to mice receiving sham stimulation [2]. These results were extended by Koopman et al. findings demonstrating significantly reduced serum levels of pro-inflammatory cytokines TNF, IL-1 $\beta$, and IL- 6 after 42 days of VNS in humans [11]. Promisingly, patients receiving VNS demonstrated significantly improved RA disease severity, including patients with treatment-refractory RA.

Murine models of IBD have also demonstrated the important role of the autonomic nervous system in the disease, as vagotomized mice and mice receiving cholinergic antagonist therapies demonstrated increased inflammation and worsened disease, whereas treatment with cholinergic agonists decreased inflammation [12]. VNS treatment in IBD has shown similar results to RA, with mouse models demonstrating significantly reduced pro-inflammatory markers with VNS [13, 14]. VNS mice also demonstrated 13-fold diminished body weight loss, an IBD disease severity marker. Studies for VNS for IBD in humans are ongoing, with initial results looking promising; 5 of 7 patients receiving 6 months of VNS demonstrated decreased disease activity, serum CRP levels, and improved vagal tone as measured by heartrate variability [15].

\section{DISCUSSION}

Current therapies for inflammatory skin disease, such as topical steroids, seek to directly reduce inflammation at the end organ-the skin. However, more generalized disease often requires treatment with systemic anti-inflammatory action, such as with biologics, immunosuppressants, or oral steroids. TNF- $\alpha$ inhibitors are currently approved for RA, PsA, psoriasis, hidradenitis suppurativa, IBD, ankylosing spondylitis, juvenile idiopathic arthritis, and uveitis, but are sometimes used off-label for other recalcitrant inflammatory skin diseases, including pityriasis rubra pilaris and Behçet's disease [16].

Increased neuronal density has been observed in skin affected by chronic inflammatory skin disease $[17,18]$, and denervation of these areas has been shown to result in disease remission in psoriasis [19]. Additionally, stress is a common trigger for inflammatory skin disease [20], and behavioral and psychological interventions can reduce disease severity in these patients [21, 22]. Thus, the nervous system 
likely plays an important role in the pathogenesis of inflammatory skin disease.

Cholinergic stimulation significantly decreases production of pro-inflammatory cytokines TNF, IL-1 $\beta$, and IL-6 [2, 11], a response profile similarly seen with successful TNF- $\alpha$ treatment in psoriasis patients [23]. As TNF- $\alpha$ inhibitors have shown efficacy for both on-label and off-label use for various inflammatory skin diseases [16], therapies inducing similar cytokine profile changes may reduce disease severity in these same diseases. Thus, we postulate that neuromodulation could work similarly to TNF- $\alpha$ inhibitors to interrupt chronic inflammation, and potentially treat inflammatory skin disease.

Breakthroughs in the importance of parasympathetic tone in the inflammatory response have highlighted the role of the nervous system in maintaining proper immune function. The known autonomic imbalances (sympathetic $>$ parasympathetic) seen in inflammatory disease give credence to the inflammatory reflex being an integral part of these diseases' pathogenesis, a theory supported by the efficacy of VNS in reducing RA and IBD disease severity.

Historically, treatments for dermatologic diseases have often come from treatments for other disease. Mainstays in psoriasis treatment, such as adalimumab, etanercept, and infliximab, have all come after initial use of these drugs in RA. Thus, the promising results of neuromodulation in RA and IBD can open up an exciting new realm of possibilities for novel treatments for inflammatory dermatologic conditions. With a proven favorable long-term safety profile for its current clinical indications of epilepsy, cluster headaches, and major depression, VNS could offer an optimal treatment approach for patients with recalcitrant inflammatory skin disease.

Although neuromodulation has shown efficacy in other inflammatory diseases, it has not yet been seriously explored in dermatology. Further exploration into this promising field will help us increase our understanding of these burdensome chronic diseases, and improve the treatment that we provide for our patients.

\section{ACKNOWLEDGEMENTS}

Funding. No funding was received for the preparation of this commentary. Article processing charges were funded by the author.

Authorship. All named authors meet the International Committee of Medical Journal Editors (ICMJE) criteria for authorship for this article, take responsibility for the integrity of the work as a whole, and have given their approval for this version to be published.

Disclosures. Dr. Koo has received honoraria from Janssen, Eli-Lilly, Celgene, AbbVie, and Leo Pharma. Eric J Yang, Sahil Sekhon, Kristen $\mathrm{M}$ Beck, and Tina Bhutani have nothing to disclose.

Compliance with Ethics Guidelines. The article is based on previously conducted studies and does not contain any studies with human participants or animals performed by any of the authors.

Open Access. This article is distributed under the terms of the Creative Commons Attribution-NonCommercial 4.0 International License (http://creativecommons.org/licenses/ by-nc/4.0/), which permits any noncommercial use, distribution, and reproduction in any medium, provided you give appropriate credit to the original author(s) and the source, provide a link to the Creative Commons license, and indicate if changes were made.

\section{REFERENCES}

1. Tracey KJ. Reflex control of immunity. Nat Rev Immunol. 2009;9(6):418-28.

2. Borovikova LV, Ivanova $S$, Zhang $M$, et al. Vagus nerve stimulation attenuates the systemic inflammatory response to endotoxin. Nature. 2000;405(6785):458-62.

3. Lindgren S, Lilja B, Rosen I, Sundkvist G. Disturbed autonomic nerve function in patients with Crohn's disease. Scand J Gastroenterol. 1991;26(4):361-6. 
4. Lindgren S, Stewenius J, Sjolund K, Lilja B, Sundkvist G. Autonomic vagal nerve dysfunction in patients with ulcerative colitis. Scand J Gastroenterol. 1993;28(7):638-42.

5. Koopman FA, Tang MW, Vermeij J, et al. Autonomic dysfunction precedes development of rheumatoid arthritis: a prospective cohort study. EBioMedicine. 2016;6:231-7.

6. Altawil R, Kadetoff D, Westman M, et al. A proposed mechanism for autonomic dysfunction in rheumatoid arthritis-reduced vagal activity related to high intrathecal IL-1 $\beta$ levels. Ann Rheum Dis. 2012;71(1):7.

7. Syngle A, Verma I, Garg N, Krishan P. Autonomic dysfunction in psoriatic arthritis. Clin Rheumatol. 2013;32(7):1059-64.

8. Holman AJ, Ng E. Heart rate variability predicts anti-tumor necrosis factor therapy response for inflammatory arthritis. Auton Neurosci. 2008;143(1-2):58-67.

9. van Maanen MA, Papke RL, Koopman FA, et al. Two novel alpha7 nicotinic acetylcholine receptor ligands: in vitro properties and their efficacy in collagen-induced arthritis in mice. PLoS One. 2015;10(1):e0116227.

10. van Maanen MA, Lebre MC, van der Poll T, et al. Stimulation of nicotinic acetylcholine receptors attenuates collagen-induced arthritis in mice. Arthritis Rheum. 2009;60(1):114-22.

11. Koopman FA, Chavan SS, Miljko S, et al. Vagus nerve stimulation inhibits cytokine production and attenuates disease severity in rheumatoid arthritis. Proc Natl Acad Sci USA. 2016;113(29):8284-9.

12. Ghia JE, Blennerhassett $\mathrm{P}$, Kumar-Ondiveeran $\mathrm{H}$, Verdu EF, Collins SM. The vagus nerve: a tonic inhibitory influence associated with inflammatory bowel disease in a murine model. Gastroenterology. 2006;131(4):1122-30.

13. Meregnani J, Clarencon D, Vivier M, et al. Anti-inflammatory effect of vagus nerve stimulation in a rat model of inflammatory bowel disease. Auton Neurosci. 2011;160(1-2):82-9.
14. Sun $P$, Zhou $K$, Wang $S$, et al. Involvement of MAPK/NF-kappaB signaling in the activation of the cholinergic anti-inflammatory pathway in experimental colitis by chronic vagus nerve stimulation. PLoS one. 2013;8(8):e69424.

15. Bonaz B, Sinniger V, Hoffmann D, et al. Chronic vagus nerve stimulation in Crohn's disease: a 6-month follow-up pilot study. Neurogastroenterol Motil. 2016;28(6):948-53.

16. Alexis AF, Strober BE. Off-label dermatologic uses of anti-TNF-a therapies. J Cutan Med Surg. 2005;9(6):296-302.

17. Nakamura M, Toyoda M, Morohashi M. Pruritogenic mediators in psoriasis vulgaris: comparative evaluation of itch-associated cutaneous factors. Br J Dermatol. 2003;149(4):718-30.

18. Tobin D, Nabarro G, de la Faille HB, van Vloten WA, van der Putte SC, Schuurman HJ. Increased number of immunoreactive nerve fibers in atopic dermatitis. J Allergy Clin Immunol. 1992;90(4):613-22.

19. Zhu TH, Nakamura M, Farahnik B, et al. The role of the nervous system in the pathophysiology of psoriasis: a review of cases of psoriasis remission or improvement following denervation injury. Am J Clin Dermatol. 2016;17(3):257-63.

20. Huynh M, Gupta R, Koo JY. Emotional stress as a trigger for inflammatory skin disorders. Semin Cutan Med Surg. 2013;32(2):68-72.

21. Tran AN, Koo JY. Evaluating the effectiveness of psychological interventions in patients with psoriasis: a review. Psoriasis Forum. 2014;20(1):8-14.

22. Ehlers A, Stangier U, Gieler U. Treatment of atopic dermatitis: a comparison of psychological and dermatological approaches to relapse prevention. J Consult Clin Psychol. 1995;63(4):624-35.

23. Balato A, Schiattarella M, Di Caprio R, et al. Effects of adalimumab therapy in adult subjects with moderate-to-severe psoriasis on Th17 pathway. J Eur Acad Dermatol Venereol. 2014;28(8):1016-24. 